Mini Review

\title{
Cervical epidural blockade: a technique in disuse or alternative to other techniques
}

\begin{abstract}
Summary
The cervical epidural blockade is a procedure that little is done through fear, ignorance and lack of training in medical units residences. Anatomy is not very different from other neuraxial areas, which gives a similar especially lumbar approach. Complications are rare, knowing the changes in various systems; it is possible to perform in expert hands as surely a lumbar epidural block from the technical point of view. Convenient serious studies and compare different techniques of regional anesthesia to assess risk, comfort and cost.
\end{abstract}

Keywords: brachial plexus, cervical epidural block, phrenic nerve, respiratory distress, hematoma, cardiovascular effects
Volume 4 Issue I - 2016

Juan Ignacio Arce Sanchez

Hospital Angeles Culiacan, Mexico

Correspondence: Juan Ignacio Arce Sanchez, Hospital Angeles Culiacan, Cd. de Hermosillo \#I2I I Col. Las Quintas, Mexico, Tel +526671428792, Email speed_jee@hotmail.com

Received: June 26, 2015 | Published: January 04, 2016

\section{Introduction}

Regional anesthesia has a wide area of choices, especially when selecting the surgical procedure to be performed, either elective or emergency. Applied neck surgeries, upper limbs (brachial plexus in its entirety) and thoracic surgery and anesthesia, analgesia or in combination with general anesthesia, depending on the needs. The cervical epidural technique is a procedure that is not regularly because of ignorance and lack practical training in medical units residence, have left for fear of complications that could be catastrophic in inexperienced hands. ${ }^{1}$ With the advent of technology tools such as ultrasound neurostimulator and they have moved this technique considered risky compared to previous ones. However, although some studies in the literature with excellent results, complications from this procedure are low are not reported., ${ }^{2,3}$ And the knowledge of anatomy and physiological changes of this technique to be well recognized even know it is much safer than others, which reports frequent complication because their approaches are: Robert M. Raw makes an exemplary description regarding the technique.

\section{Anatomy}

Very similar to the epidural space, with some differences from the processes $\mathrm{C} 5$ to $\mathrm{T} 3$ are sufficiently horizontalizadas, allowing easy approach to the midline, the prominens vertebra allows identification of C6-C7 and C7-T1, the ligamentum flavum is less developed in this region and you can usually identify a "pop" touch way to get through. The depth of the epidural space can vary from 4 to $7 \mathrm{~cm}$. The width is about $3 \mathrm{~mm}$, compared with the lumbar region of 5-6 $\mathrm{mm}$.

Venous plexus are well developed in the side and less expensive in the posterior midline, without valves and connecting up to the brain sinuses, par vertebral veins below central flow, a small dose of local anesthetic in these veins may cause seizures snapshots. Indications and uses involve any proceeding involving blocking nerve roots $\mathrm{C} 3$ to T6 whether anesthesia or analgesia.

Contraindications are the same as for the lumbar epidural block: the denial of art, infections near the site blocking, coagulopathy, limited cardiac output, lack of experience in epidural anesthesia (this lock is not for beginners) and inability to revive or general anesthesia. Serious complications and sequelae are few and have been described in the scientific literature. The idea of such complications is very rare, partly because of the high skill training and anesthesiologists who perform this procedure (Figure 1)

\section{Theoretical complications}

Difficulty breathing is thought that turning this procedure at this point both phrenic nerves were blocked by disabling ventilation, however, seems to be associated with sympathetic nerve block. Studies have confirmed their safety with little effect on ventilation, except for patients with pulmonary disability, with no fatalities arterial blood gas concern.

Considering that all regional techniques have the possibility of peripherally phrenic nerve block or impair ventilation. It could be considered that this technique is the safest in the surgical area. You need to study the reasons which does not happen respiratory failure in this block, we know that may be due to several factors: As a segmental dermatomes simply lock neck and thoracic region, keeping the mechanics of the intercostals muscles and adequate tidal volumes, even if they have bilateral phrenic nerve block. Clearly there for breathing by blocking the intercostal nerves and the phrenic nerve. The phrenic nerve is more resistant than other motor blockade motor nerves, making it unlikely blocking, in consideration of the interscalene block, where the most often of blocks, should be considered a comparative study on this scenario.

The motor roots is in the anterior region and has been suggested as the approach is made in the posterior region, the epidural space is broader and is selectively diffused into the posterior roots that are more sensitive, and it is said that the ligament the epidural space in the lateral position epidural prevents diffusion of the drugs to the motor roots. Added to this the physicochemical characteristics of local anesthetics such as ropivacaine, which is more sensitive than motor action and the concentrations of other anesthetics such as lidocaine and bupivacaine, this will allow selective effectiveness of their mechanisms of action.

The cervical hematoma is rarely disclosed and described in patients who are undergoing pain management with steroid injection. It may be by the approach, as it is done so paravertebral and greater vascular supply is in the sides of the vertebral bodies. It is considered less risky than the lumbar region and the neck venous pressure is lower in the sitting position of the patient. The epidural abscess is rarely reported in the same way. Rarely hypotension bradycardia requiring management antimuscarinic show, which responds quite well, hypotension occurs much less than the lumbar blockages due to the preservation of the sympathetic tone of the viscera and lower extremities. 

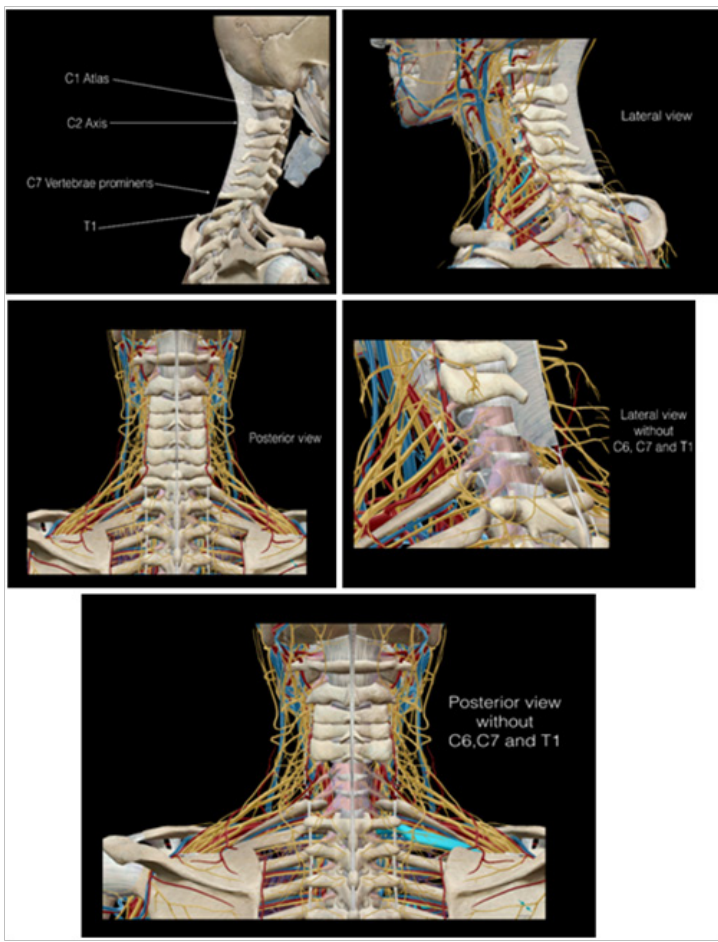

Figure I Lateral and posterior view with $\mathrm{C} 6, \mathrm{C} 7$ and TI.

Direct spinal cord injury is a little complication and has been described in patients operated for chronic pain with application of steroids in which attribute to flouroscopia misused and sedation decreased the possibility of referring symptoms. It is here that one wonders if they really epidural blocks must be done under sedation or not? There are studies that relate the incidence of puncture is $0.7 \%$, $0.3 \%$ radicular pain that gave the withdrawal of the catheter. The epidural catheter placement above the spinal cord is a safe practice in reference to the possibility of spinal cord injury and clinical possibility of blockages cervical epidural, and puncturing the cord despite being uncomfortable for the patient and the physician does not result in permanent damage, since most cases of injury they reported were to THROUGH needle injection of potentially harmful drugs such as steroids, that are due to apply medicine THROUGH catheter previously easily introduced and after negative aspiration in which there should be no blood or cerebrospinal fluid and any other lock epidural at different levels.

\section{Cardiovascular effects on TI-T4}

It has been shown that the sympathetic innervation of these fibers and stimulates chronotropy inotropy and blocking these fibers leads to severe bradycardia. The benchmark for hemodynamic changes are mentioned in the book "Neural blockade" of M Cousins apparently it reduces this blocking $80 \%$ of the sympathetic effect and that the net effect will be to:

a. Splanchnic fibers are locked (T6-L1).

b. Vasoconstrictor fibers of the lower limbs (T11-L4).

c. Intravascular volume depleted.

d. Systemic absorption of local anesthetics (myocardial depression).

e. Parasympathetic hyperactivity at the same time., ${ }^{4,5}$

\section{Technique}

\section{Position}

The best position is sitting, his neck bent head, this allows the alignment of the vertebrae, better exposure of the processes and the intervertebral spaces. Identification of space: it can be with the technique of the hanging drop or loss of strength with saline, use of air could cause pain for the likely migration into the ventricles and make Pneumocephalus (Figure 2).
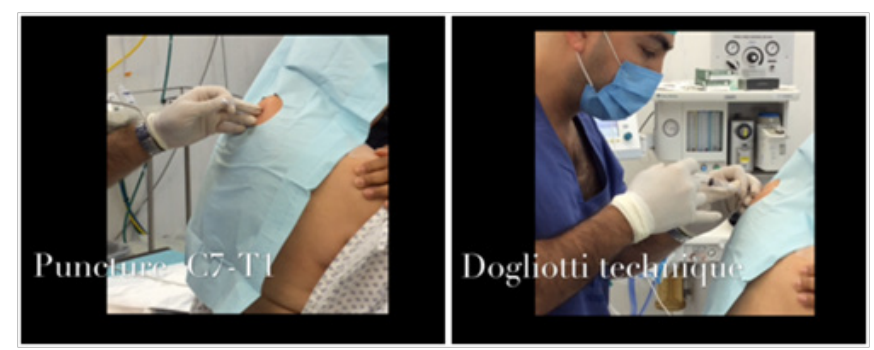

Catheter: The catheter should be no resistance and 3-5 cm into the epidural space.
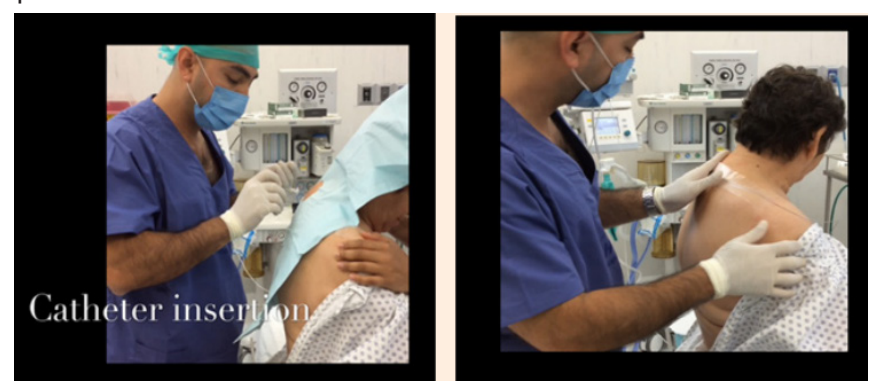

Dose: As suggested $0.5 \mathrm{ml}$ volume segment block, type of anesthesia and the concentration will give the anesthetic or analgesic effect according to needs.

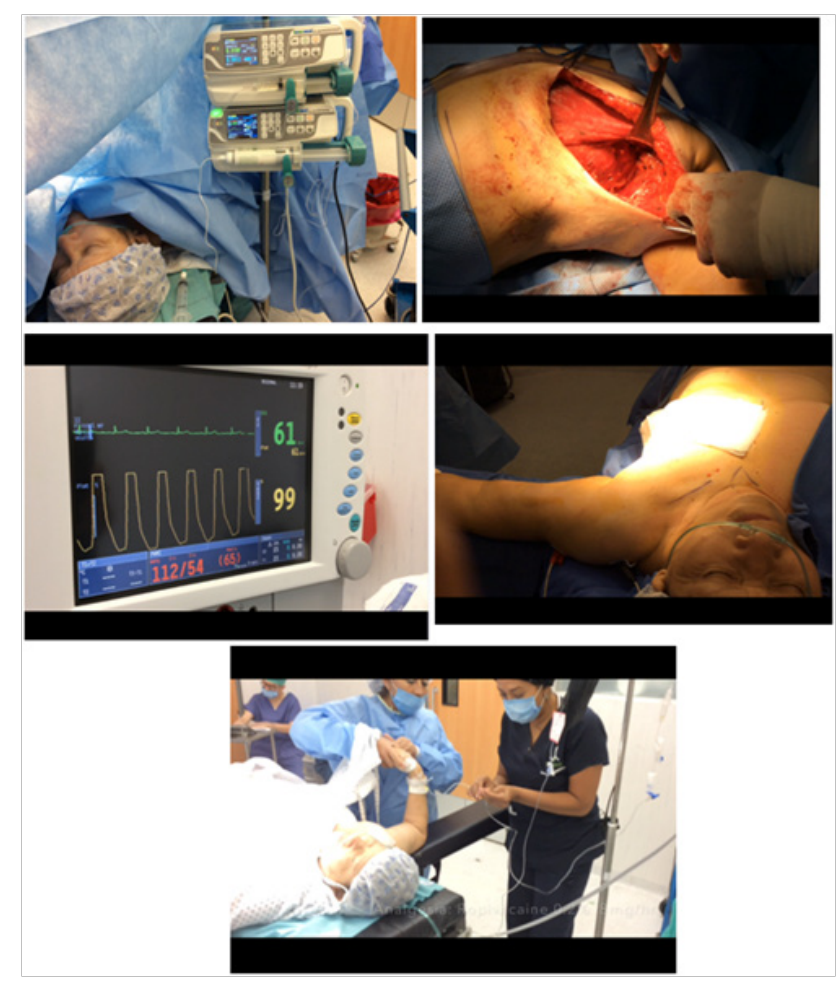

Figure 2 Technique. 


\section{Conclusion}

Certainlyitis concluded that in clinical practice few anesthesiologists who perform this procedure due to the lack of practice, confidence and ability to make blocks above the lumbar spaces. However you do this type of locks knows the indulgent experience for both the patient and the anesthesiologist and perioperative team. That the application of this technique is much easier, faster and safer, as well as catheter placement for continuous, predictable and secure infusions compared with an interscalene, supraclavicular, infraclavicular, axillary and paravertebral block, including ultrasound-guided. It is possible that in the future can be made comparative studies to measure levels of satisfaction and effectiveness with respect to cervical epidural block this I would say, in most medical centers obsolete world. ${ }^{6,7}$

\section{Acknowledgments}

None.

\section{Conflicts of Interset}

None.

\section{References}

1. Shawn E, Banks, Sharp. Curr Opinion in Anesthesiology. 2015;28(2):201-205.

2. Dominguez F, Laso T, Tijero T, et al. Cervical epidural anesthesia with $0.75 \%$ ropivacaine in shoulder surgery. Rev Esp Anestesiol Reanim. 2002;49(1):39-43.

3. LJ Mejia. Mexican Anesthesiology Journal. 2013;36(suppl1).

4. Raw Robert MD. Book 8-2 PAC Anesthesia Regional anesthesia and postoperative pain Part I. Latin American Edition, USA, 2002. p. 69-77.

5. Ramsin Benyamin, Vijay Singh, Allan T, et al. Systematic Review of the Effectiveness of Cervical Epidurals in the Management of Chronic Neck Pain. Pain Physician. 2009;12:137-157.

6. Manchikanti L, Boswell MV, Singh V, et al. Comprehensive evidencebased guidelines for interventional techniques in the management of chronic spinal pain. Pain Physician. 2009;12(4):699-802.

7. Kevin King, Jacques E. Para vertebral Blocks: The Evolution of a Standard of Care. Anesthesiology News March. 2012. 